\title{
A mean field theory of coded CDMA systems
}

\author{
Toru Yano ${ }^{1}$, Toshiyuki Tanaka ${ }^{2}$ and David Saad ${ }^{3}$ \\ ${ }^{1}$ Graduate School of Science and Technology, Keio University, Hiyoshi, Kohoku-ku, \\ Yokohama-shi, Kanagawa 223-8522, Japan \\ ${ }^{2}$ Graduate School of Informatics, Kyoto University, Yoshida Hon-machi, Sakyo-ku, \\ Kyoto-shi, Kyoto 606-8501, Japan \\ ${ }^{3}$ Neural Computing Research Group, Aston University, Birmingham B4 7ET, UK \\ E-mail: yano@thx.appi.keio.ac.jp
}

\begin{abstract}
We present a mean field theory of code-division multiple-access (CDMA) systems with error-control coding. On the basis of the relation between the free energy and mutual information, we obtain an analytical expression of the maximum spectral efficiency of the coded CDMA system, from which a mean-field description of the coded CDMA system is provided in terms of a bank of scalar Gaussian channels whose variances in general vary at different code symbol positions. Regular low-density paritycheck (LDPC)-coded CDMA systems are also discussed as an example of the coded CDMA systems.
\end{abstract}

PACS numbers: 89.70.-a, 75.10.Nr, 84.40.Ua

\section{Introduction}

In modern societies, many information technology-based applications, such as cellular telephony networks, require unconstrained connectivity, which rely heavily on wireless communications. In recent years, spin glass theory has been applied to analysis of wireless communication systems, such as code-division multiple-access (CDMA) and multiple-input multiple-output (MIMO) systems, by mapping these systems onto disordered spin systems [1-6].

Replica analysis has successfully provided quantitative characterizations of these wireless communication systems in terms of single-body mean-field descriptions. Guo and Verdú [3], extending analysis of Tanaka [1,2], have claimed that a randomly-spread CDMA channel, where users modulate their symbols with randomly generated spreading sequences, is statistically equivalent to a bank of scalar Gaussian channels in the largesystem (thermodynamical) limit. Their claim, which they termed the "decoupling principle," can be regarded as one form of mean field theory. It is nevertheless of particular significance, because the resulting mean-field description, which gives a representation of a complex communication system in terms of scalar Gaussian channels, makes sense from a communication theory point of view. 
Most existing studies discussed uncoded CDMA systems, in which error-control coding is not explicitly taken into account. On the other hand, real-world CDMA-based systems, such as the third-generation cellular phone systems, are equipped with errorcontrol coding in order to achieve high-performance communication [7]. In this paper, we study a mean field theory of coded CDMA systems, in order to obtain explicitly their mean-field descriptions, similar to those of uncoded systems, and to see whether the derived mean-field formulation provides a simplified representations of coded CDMA systems in terms of simple communication systems.

This paper is organized as follows. In section 2 we introduce the coded CDMA channel model to be analyzed. In section 3 we present a mean field theory of the model. On the basis of a relation between the mutual information of the channel model and the free energy of the system [8], we show a mean-field description of the coded CDMA systems. In section 4, we discuss regular LDPC-coded CDMA systems as an example of coded CDMA systems, followed by a conclusions section.

\section{System model and multiuser detection}

We consider an uplink of a randomly-spread coded CDMA system with $K$ users. User $k$ encodes an original message $\boldsymbol{\xi}_{k}$ into a codeword $\boldsymbol{c}_{k}=\left(c_{k}^{i}\right)$ with $c_{k}^{i}= \pm 1$, with an encoding function $g_{k}(\cdot)$

$$
\boldsymbol{c}_{k}=g_{k}\left(\boldsymbol{\xi}_{k}\right) \text {. }
$$

We assume that codelengths $M$ are the same for all users.

Codewords are modulated with spreading sequences, and then transmitted to a receiver. The spreading sequence used to modulate $i$ th symbol of the codeword of $k$ th user, $c_{k}^{i}$, is denoted by $\boldsymbol{s}_{k}^{i}=\frac{1}{\sqrt{W}}\left(s_{1 k}^{i}, \ldots, s_{W k}^{i}\right)$, for which we assume random spreading, meaning that $s_{\mu k}^{i}$ 's are independent and identically-distributed (i.i.d.) realvalued random variables with zero mean, unit variance and finite higher-order moments. The received signals at the receiver through the CDMA channel are given by

$$
y_{\mu}^{i}=\frac{1}{\sqrt{W}} \sum_{k=1}^{K} A_{k} c_{k}^{i} s_{\mu k}^{i}+n_{\mu}^{i} \quad(i=1, \ldots, M ; \mu=1, \ldots, W),
$$

where $A_{k} \in \mathbb{R}$ denotes an amplitude of user $k$ 's signal, representing attenuation (fading) effects, and where $n_{\mu}^{i}$ 's are real-valued additive noise, which are assumed i.i.d. random variables following a probability distribution $\rho_{0}(\cdot)$. Equation $(2)$ is simplified by letting $x_{k}^{i}=A_{k} c_{k}^{i}$ :

$$
y_{\mu}^{i}=\frac{1}{\sqrt{W}} \sum_{k=1}^{K} x_{k}^{i} s_{\mu k}^{i}+n_{\mu}^{i} \quad(i=1, \ldots, M ; \mu=1, \ldots, W) .
$$

In the present paper we follow the Bayesian framework. By letting $\boldsymbol{x}_{k}=$ $\left(x_{k}^{1}, \ldots, x_{k}^{M}\right)$ and $\boldsymbol{y}_{\mu}=\left(y_{\mu}^{1}, \ldots, y_{\mu}^{M}\right)$, the entire channel input and output are denoted by $\overrightarrow{\boldsymbol{x}}=\left(\boldsymbol{x}_{1}, \ldots, \boldsymbol{x}_{K}\right)$ and $\overrightarrow{\boldsymbol{y}}=\left(\boldsymbol{y}_{1}, \ldots, \boldsymbol{y}_{W}\right)$, respectively. From the above-mentioned setting, all information of the users' encoding schemes as well as the channel attenuation 
are encapsulated in a prior distribution $p_{0}(\overrightarrow{\boldsymbol{x}})$ of the entire channel input $\overrightarrow{\boldsymbol{x}}$. The receiver postulates that the prior distribution of the channel input is $p(\overrightarrow{\boldsymbol{x}})$ and that the distribution of the noise is $\rho(\cdot)$. The true and postulated distributions of $\overrightarrow{\boldsymbol{y}}$ conditioned on $\overrightarrow{\boldsymbol{x}}$ are thus given by

$$
\begin{aligned}
& p_{0}(\overrightarrow{\boldsymbol{y}} \mid \overrightarrow{\boldsymbol{x}})=\prod_{i=1}^{M} \prod_{\mu=1}^{W} \rho_{0}\left(y_{\mu}^{i}-\frac{1}{\sqrt{W}} \sum_{k=1}^{K} s_{\mu k}^{i} x_{k}^{i}\right) \quad \text { and } \\
& p(\overrightarrow{\boldsymbol{y}} \mid \overrightarrow{\boldsymbol{x}})=\prod_{i=1}^{M} \prod_{\mu=1}^{W} \rho\left(y_{\mu}^{i}-\frac{1}{\sqrt{W}} \sum_{k=1}^{K} s_{\mu k}^{i} x_{k}^{i}\right),
\end{aligned}
$$

respectively. The receiver has to infer $\overrightarrow{\boldsymbol{x}}$ from $\overrightarrow{\boldsymbol{y}}$, which is called multiuser detection. On the basis of the Bayesian framework, multiuser detection is formulated as a statistical inference problem of $\overrightarrow{\boldsymbol{x}}$ from $\overrightarrow{\boldsymbol{y}}$. A broad range of multiuser detectors [9], including optimal and linear ones, can be constructed by the generalized posterior mean estimator (GPME) $[2,3]$

$$
\left[\boldsymbol{x}_{k}\right]=\int \boldsymbol{x}_{k} p(\overrightarrow{\boldsymbol{x}} \mid \overrightarrow{\boldsymbol{y}}) \mathrm{d} \overrightarrow{\boldsymbol{x}},
$$

where $p(\overrightarrow{\boldsymbol{x}} \mid \overrightarrow{\boldsymbol{y}})$ is the posterior distribution postulated by the receiver, given by

$$
p(\overrightarrow{\boldsymbol{x}} \mid \overrightarrow{\boldsymbol{y}})=\frac{p(\overrightarrow{\boldsymbol{y}} \mid \overrightarrow{\boldsymbol{x}}) p(\overrightarrow{\boldsymbol{x}})}{\pi(\overrightarrow{\boldsymbol{y}})},
$$

with $\pi(\overrightarrow{\boldsymbol{y}})=\int p(\overrightarrow{\boldsymbol{x}}) p(\overrightarrow{\boldsymbol{y}} \mid \overrightarrow{\boldsymbol{x}}) \mathrm{d} \overrightarrow{\boldsymbol{x}}$ being the postulated marginal distribution of $\overrightarrow{\boldsymbol{y}}$. The posterior distribution $p(\overrightarrow{\boldsymbol{x}} \mid \overrightarrow{\boldsymbol{y}})$ can alternatively be represented as a Boltzmann distribution:

$$
p(\overrightarrow{\boldsymbol{x}} \mid \overrightarrow{\boldsymbol{y}}) \propto \exp (-\gamma \mathcal{H}(\overrightarrow{\boldsymbol{x}})),
$$

with the Hamiltonian

$$
\mathcal{H}(\overrightarrow{\boldsymbol{x}})=-\sum_{i, \mu} \log \rho\left(y_{\mu}^{i}-\frac{1}{\sqrt{W}} \sum_{k=1}^{K} s_{\mu k}^{i} x_{k}^{i}\right)-\log p(\overrightarrow{\boldsymbol{x}}),
$$

and where the inverse temperature is $\gamma=1$. Accordingly, GPME $\left[\boldsymbol{x}_{k}\right]$ can be regarded as a site magnetization of the system characterized by the Hamiltonian $\mathcal{H}(\overrightarrow{\boldsymbol{x}})$. Note that in this system each "spin" variable, $\boldsymbol{x}_{k}$, is a collection of $M$ variables, each of which corresponds to a codeword symbol with fading effects.

\section{Mean field theory}

\subsection{Mutual information and free energy}

Mutual information [10] quantifies theoretical information transmission capabilities of a given channel model. We define a generalized mutual information $I(\overrightarrow{\boldsymbol{X}} ; \overrightarrow{\boldsymbol{Y}})$ of the introduced model as

$$
I(\overrightarrow{\boldsymbol{X}} ; \overrightarrow{\boldsymbol{Y}})=\int p_{0}(\overrightarrow{\boldsymbol{x}}) \int p_{0}(\overrightarrow{\boldsymbol{y}} \mid \overrightarrow{\boldsymbol{x}}) \log \frac{p(\overrightarrow{\boldsymbol{y}} \mid \overrightarrow{\boldsymbol{x}})}{\pi(\overrightarrow{\boldsymbol{y}})} \mathrm{d} \overrightarrow{\boldsymbol{y}} \mathrm{d} \overrightarrow{\boldsymbol{x}},
$$


where, and hereafter, logarithms are taken to base $e$. The conventional definition of mutual information, denoted by $I_{0}(\overrightarrow{\boldsymbol{X}} ; \overrightarrow{\boldsymbol{Y}})$, is recovered if we let $p(\overrightarrow{\boldsymbol{y}} \mid \overrightarrow{\boldsymbol{x}})=p_{0}(\overrightarrow{\boldsymbol{y}} \mid \overrightarrow{\boldsymbol{x}})$ and $p(\overrightarrow{\boldsymbol{x}})=p_{0}(\overrightarrow{\boldsymbol{x}})$ in $(10)$. The spectral efficiency (an upper limit of data rates, divided by bandwidth, where reliable communication is possible) [11], which is also a performance measure frequently used in the context of wireless communication, is defined as

$$
\mathrm{C}=\frac{1}{W} I(\overrightarrow{\boldsymbol{X}} ; \overrightarrow{\boldsymbol{Y}})
$$

The generalized mutual information can be decomposed as

$$
I(\overrightarrow{\boldsymbol{X}} ; \overrightarrow{\boldsymbol{Y}})=H(\overrightarrow{\boldsymbol{Y}})-H(\overrightarrow{\boldsymbol{Y}} \mid \overrightarrow{\boldsymbol{X}})
$$

where

$$
H(\overrightarrow{\boldsymbol{Y}})=-\int \pi_{0}(\overrightarrow{\boldsymbol{y}}) \log \pi(\overrightarrow{\boldsymbol{y}}) \mathrm{d} \overrightarrow{\boldsymbol{y}}
$$

is the (Shannon) differential entropy of $\overrightarrow{\boldsymbol{y}}$, where the true marginal distribution of $\overrightarrow{\boldsymbol{y}}$ is $\pi_{0}(\overrightarrow{\boldsymbol{y}})=\int p_{0}(\overrightarrow{\boldsymbol{x}}) p_{0}(\overrightarrow{\boldsymbol{y}} \mid \overrightarrow{\boldsymbol{x}}) \mathrm{d} \overrightarrow{\boldsymbol{x}}$, and where

$$
H(\overrightarrow{\boldsymbol{Y}} \mid \overrightarrow{\boldsymbol{X}})=-\int p_{0}(\overrightarrow{\boldsymbol{x}}) \int p_{0}(\overrightarrow{\boldsymbol{y}} \mid \overrightarrow{\boldsymbol{x}}) \log p(\overrightarrow{\boldsymbol{y}} \mid \overrightarrow{\boldsymbol{x}}) \mathrm{d} \overrightarrow{\boldsymbol{y}} \mathrm{d} \overrightarrow{\boldsymbol{x}}
$$

is the conditional differential entropy of $\overrightarrow{\boldsymbol{y}}$ conditioned on $\overrightarrow{\boldsymbol{x}}$, which is equal to the differential entropy of the channel noise. By noticing that $-\log \pi(\overrightarrow{\boldsymbol{y}})$ is the free energy of the system characterized by the posterior distribution $p(\overrightarrow{\boldsymbol{x}} \mid \overrightarrow{\boldsymbol{y}})$, we are encouraged to adopt statistical mechanical approaches to evaluate $H(\overrightarrow{\boldsymbol{Y}})$, which is the free energy averaged with respect to the received signals. The relation between the mutual information and the free energy (averaged over the received signals) was pointed out explicitly in [8].

We consider the large-system limit, where $K$ and $W$ go to infinity with the load $\beta=K / W$ fixed, and the random spreading, in which one has to evaluate $H(\overrightarrow{\boldsymbol{Y}} \mid S)$, where $S=\left\{s_{\mu k}^{i}\right\}$ denotes the collection of the spreading sequences. Assuming the selfaveraging property for $H(\overrightarrow{\boldsymbol{Y}} \mid S)$, we evaluate, in the large-system limit, the quantity:

$$
h=\lim _{K \rightarrow \infty} \frac{1}{K} H(\overrightarrow{\boldsymbol{Y}} \mid S) .
$$

We evaluate $h$ using the replica method. We first rewrite (15) as

$$
h=-\lim _{K \rightarrow \infty} \frac{1}{K} \lim _{n \rightarrow 0} \frac{\partial}{\partial n} \log \Xi_{n}=-\lim _{n \rightarrow 0} \frac{\partial}{\partial n} \lim _{K \rightarrow \infty} \frac{1}{K} \log \Xi_{n},
$$

where

$$
\Xi_{n}=\int \mathrm{E}_{S}\left\{\pi_{0}(\overrightarrow{\boldsymbol{y}})[\pi(\overrightarrow{\boldsymbol{y}})]^{n}\right\} \mathrm{d} \overrightarrow{\boldsymbol{y}}
$$

with $\mathrm{E}_{S}\{\cdot\}$ denoting averaging with respect to the spreading sequences $S$. We evaluate $\lim _{K \rightarrow \infty} K^{-1} \log \Xi_{n}$ in the large-system limit, assuming that $n$ is a positive integer, and then extend the result to real values of $n$ in the vicinity of 0 to obtain $h$. 
Following the replica analysis of $[2,3]$, we obtain, under the assumption of replica symmetry (RS) (see Appendix A for the derivation),

$$
\begin{aligned}
& h=-\beta^{-1} \sum_{i=1}^{M} \int \bar{\rho}_{0}\left(y^{i}-\sqrt{\frac{\beta\left(m^{i}\right)^{2}}{q^{i}}} t^{i}\right) \log \left[\bar{\rho}\left(y^{i}-\sqrt{\beta q^{i}} t^{i}\right)\right] D t^{i} \mathrm{~d} y^{i} \\
& +\sum_{i}\left\{r^{i} \frac{F^{i}-E^{i}}{2}+m^{i} E^{i}-\frac{1}{2} q^{i} F^{i}-\frac{F^{i}}{2 E^{i}}-\frac{E^{i}}{2} r_{0}^{i}-\frac{1}{2} \log \frac{2 \pi}{E^{i}}\right\} \\
& -\lim _{K \rightarrow \infty} \frac{1}{K}\left\langle\left\langle\log \int p(\overrightarrow{\boldsymbol{x}}) \prod_{k} \rho_{G}\left(\boldsymbol{z}_{k} \mid \boldsymbol{x}_{k}\right) \mathrm{d} \overrightarrow{\boldsymbol{x}}\right\rangle\right\rangle,
\end{aligned}
$$

where $\bar{\rho}_{0}(\cdot)$ and $\bar{\rho}(\cdot)$ are defined as

$$
\begin{aligned}
& \bar{\rho}_{0}\left(w^{i}\right)=\int \rho_{0}\left(w^{i}-\sqrt{\beta\left(r_{0}^{i}-\frac{\left(m^{i}\right)^{2}}{q^{i}}\right)} n\right) D n \\
& \bar{\rho}\left(w^{i}\right)=\int \rho\left(w^{i}-\sqrt{\beta\left(r^{i}-q^{i}\right)} n\right) D n
\end{aligned}
$$

with $D n=e^{-n^{2} / 2} \mathrm{~d} n / \sqrt{2 \pi}$ being a Gaussian measure, where $\rho_{G 0}\left(\boldsymbol{z}_{k} \mid \boldsymbol{x}_{0 k}\right)$ and $\rho_{G}\left(\boldsymbol{z}_{k} \mid \boldsymbol{x}_{k}\right)$, with $\boldsymbol{z}_{k}=\left(z_{k}^{1}, z_{k}^{2}, \ldots, z_{k}^{M}\right)$, are defined as

$$
\begin{aligned}
& \rho_{G 0}\left(\boldsymbol{z}_{k} \mid \boldsymbol{x}_{0 k}\right)=\prod_{i=1}^{M}\left[\sqrt{\frac{\left(E^{i}\right)^{2}}{2 \pi F^{i}}} \exp \left\{-\frac{\left(E^{i}\right)^{2}}{2 F^{i}}\left(z_{k}^{i}-x_{0 k}^{i}\right)^{2}\right\}\right], \\
& \rho_{G}\left(\boldsymbol{z}_{k} \mid \boldsymbol{x}_{k}\right)=\prod_{i=1}^{M}\left[\sqrt{\frac{E^{i}}{2 \pi}} \exp \left\{-\frac{E^{i}}{2}\left(z_{k}^{i}-x_{k}^{i}\right)^{2}\right\}\right],
\end{aligned}
$$

and where the order parameters $\left\{E^{i}, F^{i}, r_{0}^{i}, r^{i}, m^{i}, q^{i} ; i=1, \ldots, M\right\}$ should satisfy the RS saddle-point equations

$$
\begin{aligned}
& E^{i}=\int \bar{\rho}_{0}^{\prime}\left(y^{i}-\sqrt{\frac{\beta\left(m^{i}\right)^{2}}{q^{i}}} t^{i}\right) \frac{\bar{\rho}^{\prime}\left(y^{i}-\sqrt{\beta q^{i}} t^{i}\right)}{\bar{\rho}\left(y^{i}-\sqrt{\beta q^{i}} t^{i}\right)} D t^{i} \mathrm{~d} y^{i}, \\
& F^{i}=\int \bar{\rho}_{0}\left(y^{i}-\sqrt{\frac{\beta\left(m^{i}\right)^{2}}{q^{i}}} t^{i}\right)\left(\frac{\bar{\rho}^{\prime}\left(y^{i}-\sqrt{\beta q^{i}} t^{i}\right)}{\bar{\rho}\left(y^{i}-\sqrt{\beta q^{i}} t^{i}\right)}\right)^{2} D t^{i} \mathrm{~d} y^{i}, \\
& r_{0}^{i}=\lim _{K \rightarrow \infty} \frac{1}{K}\left\langle\left\langle\left|\boldsymbol{x}_{0}^{i}\right|^{2}\right\rangle\right\rangle, \quad r^{i}=\lim _{K \rightarrow \infty} \frac{1}{K}\left\langle\left\langle\left\langle\left|\boldsymbol{x}^{i}\right|^{2}\right\rangle\right\rangle,\right. \\
& m^{i}=\lim _{K \rightarrow \infty} \frac{1}{K}\left\langle\left\langle\boldsymbol{x}_{0}^{i} \cdot\left\langle\boldsymbol{x}^{i}\right\rangle\right\rangle, \quad q^{i}=\lim _{K \rightarrow \infty} \frac{1}{K}\left\langle\left\langle\left|\left\langle\boldsymbol{x}^{i}\right\rangle\right|^{2}\right\rangle\right\rangle .\right.
\end{aligned}
$$

Here we let $\boldsymbol{x}_{0}^{i}=\left(x_{01}^{i}, \ldots, x_{0 K}^{i}\right)$ and $\boldsymbol{x}^{i}=\left(x_{1}^{i}, \ldots, x_{K}^{i}\right)$. Definitions of the symbols $\langle\cdots\rangle$ and $\langle\langle\cdots\rangle\rangle$ are

$$
\begin{aligned}
& \langle\cdots\rangle=\int(\cdots) \frac{p(\overrightarrow{\boldsymbol{x}}) \prod_{k=1}^{K} \rho_{G}\left(\boldsymbol{z}_{k} \mid \boldsymbol{x}_{k}\right)}{\int p(\overrightarrow{\boldsymbol{x}}) \prod_{k=1}^{K} \rho_{G}\left(\boldsymbol{z}_{k} \mid \boldsymbol{x}_{k}\right) \mathrm{d} \overrightarrow{\boldsymbol{x}}} \mathrm{d} \overrightarrow{\boldsymbol{x}}, \\
& \langle\langle\cdots\rangle\rangle=\int(\cdots) p_{0}\left(\overrightarrow{\boldsymbol{x}}_{0}\right) \prod_{k=1}^{K} \rho_{G 0}\left(\boldsymbol{z}_{k} \mid \boldsymbol{x}_{0 k}\right) \mathrm{d} \overrightarrow{\boldsymbol{z}} \mathrm{d} \overrightarrow{\boldsymbol{x}}_{0},
\end{aligned}
$$


respectively. One can regard the last term on the right-hand side of (18) as the per-user (Shannon) differential entropy of the outputs $\overrightarrow{\boldsymbol{z}}=\left(\boldsymbol{z}_{1}, \ldots, \boldsymbol{z}_{K}\right)$ when the codewords $\boldsymbol{c}_{k}$ are sent separately over a bank of Gaussian channels with fading, $\rho_{G 0}\left(\boldsymbol{z}_{k} \mid \boldsymbol{x}_{0 k}\right)$, $k=1, \ldots, K$, and when the receiver assumes the Gaussian channels with fading, $\rho_{G}\left(\boldsymbol{z}_{k} \mid \boldsymbol{x}_{k}\right), k=1, \ldots, K$.

The maximum spectral efficiency of the present channel model, which can be achieved by letting $p(\overrightarrow{\boldsymbol{x}})=p_{0}(\overrightarrow{\boldsymbol{x}})$ and $p(\overrightarrow{\boldsymbol{y}} \mid \overrightarrow{\boldsymbol{x}})=p_{0}(\overrightarrow{\boldsymbol{y}} \mid \overrightarrow{\boldsymbol{x}})$, is given by

$$
\begin{aligned}
& \mathrm{C}_{0}=\lim _{W \rightarrow \infty} \frac{1}{W} I_{0}(\overrightarrow{\boldsymbol{X}} ; \overrightarrow{\boldsymbol{Y}}) \\
& =-\sum_{i} \int \bar{\rho}_{0}\left(y^{i}-\sqrt{\beta q^{i}} t^{i}\right) \log \left[\bar{\rho}_{0}\left(y^{i}-\sqrt{\beta q^{i}} t^{i}\right)\right] D t^{i} \mathrm{~d} y^{i} \\
& -\frac{\beta}{2} \sum_{i}\left\{1+F^{i}\left(r^{i}-q^{i}\right)+\log \frac{2 \pi}{F^{i}}\right\}-\lim _{K \rightarrow \infty} \frac{\beta}{K}\left\langle\left\langle\log \int p_{0}(\overrightarrow{\boldsymbol{x}}) \prod_{k} \rho_{G 0}\left(\boldsymbol{z}_{k} \mid \boldsymbol{x}_{k}\right) \mathrm{d} \overrightarrow{\boldsymbol{x}}\right\rangle\right\rangle \\
& +\lim _{K \rightarrow \infty} \frac{\beta}{K} \int p_{0}(\overrightarrow{\boldsymbol{x}}) \int p_{0}(\overrightarrow{\boldsymbol{y}} \mid \overrightarrow{\boldsymbol{x}}) \log p_{0}(\overrightarrow{\boldsymbol{y}} \mid \overrightarrow{\boldsymbol{x}}) \mathrm{d} \overrightarrow{\boldsymbol{y}} \mathrm{d} \overrightarrow{\boldsymbol{x}} .
\end{aligned}
$$

This result can be regarded as an extension of Tanaka's result [12] for uncoded CDMA systems, in which each user sends one symbol, to the case where $M$ symbols are transmitted. It should be noted that the latter is not a straightforward extension of the former because in the latter we allow statistical correlations among $M$ symbols, which can arise due to coding in coded systems and are to be utilized in decoding.

\subsection{Mean field description of coded CDMA systems}

The expectations $\langle\cdots\rangle$ and $\langle\langle\cdots\rangle\rangle$, as defined in (24) and (25), respectively, have the following interpretations. Let us consider a bank of $K$ independent single-user scalar Gaussian channels, where the $k$ th user sends $M$ symbols via the $k$ th Gaussian channel, and assume that the true input-output characteristic of the whole channel usage is defined by $\prod_{k} \rho_{G 0}\left(\boldsymbol{z}_{k} \mid \boldsymbol{x}_{0 k}\right)$ and that the input-output characteristic postulated at the receiver is represented by $\prod_{k} \rho_{G}\left(\boldsymbol{z}_{k} \mid \boldsymbol{x}_{k}\right)$. Then, $\langle\cdots\rangle$ denotes the generalized posterior mean with respect to the posterior distribution of $\overrightarrow{\boldsymbol{x}}$ given channel output $\overrightarrow{\boldsymbol{z}}$, which is derived by postulating the prior distribution $p(\overrightarrow{\boldsymbol{x}})$ and the channel $\prod_{k} \rho_{G}\left(\boldsymbol{z}_{k} \mid \boldsymbol{x}_{k}\right)$. On the other hand, $\langle\langle\cdots\rangle\rangle$ is the expectation with respect to the true prior distribution $p_{0}\left(\overrightarrow{\boldsymbol{x}}_{0}\right)$ and the channel $\prod_{k} \rho_{G 0}\left(\boldsymbol{z}_{k} \mid \boldsymbol{x}_{0 k}\right)$.

Indeed, by performing replica analysis for joint moments of $\left[\boldsymbol{x}_{k}\right]$ and $\boldsymbol{x}_{0 k}$, just as in [3] (see Appendix B for detailed analysis), one can show that the joint distribution of $\left(\left[\boldsymbol{x}_{k}\right], \boldsymbol{x}_{0 k}\right)$ conditioned on the spreading sequences in the coded CDMA system converges, under random spreading and in the large-system limit, to the joint distribution of $\left(\left\langle\boldsymbol{x}_{k}\right\rangle, \boldsymbol{x}_{0 k}\right)$ in the system composed of the scalar Gaussian channels:

$$
p\left(\left[\boldsymbol{x}_{k}\right], \boldsymbol{x}_{0 k} \mid S\right) \rightarrow p\left(\left\langle\boldsymbol{x}_{k}\right\rangle, \boldsymbol{x}_{0 k}\right),
$$

which means that the decoupling principle [3] also holds for the coded CDMA system, and that the mean-field description has been obtained in terms of the single-user 


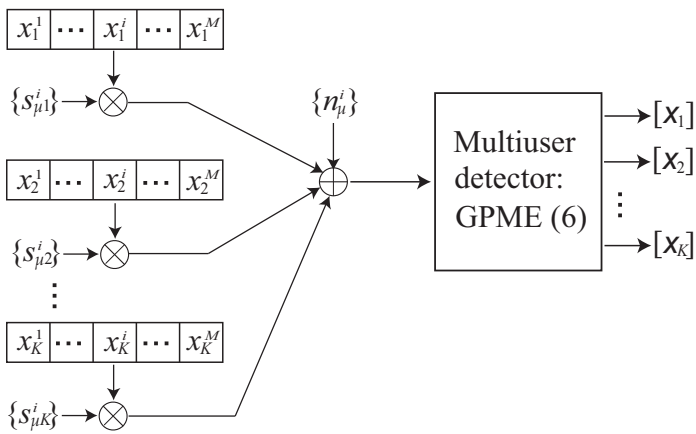

(a) CDMA multiuser channel

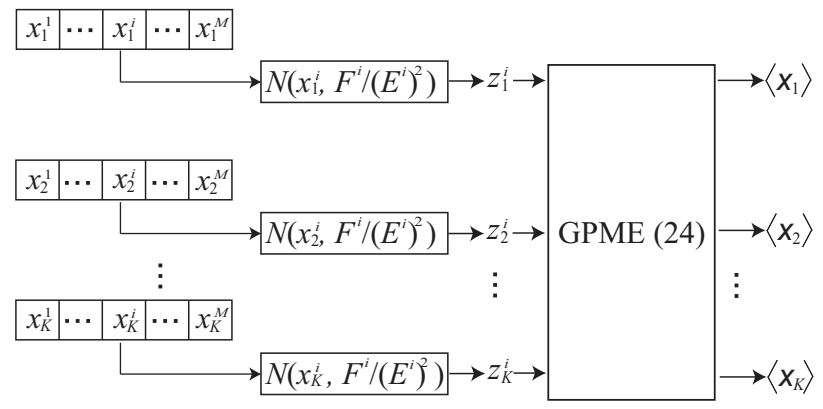

(b) A bank of scalar Gaussian channels

Figure 1. (a) Schematic representation of the coded CDMA channel. (b) Decoupling structure of the coded CDMA channel. $N\left(\mu, \sigma^{2}\right)$ denotes a Gaussian channel in which $\mu$ is a channel input and the channel noise is drawn i.i.d. from a Gaussian distribution with mean zero and variance $\sigma^{2}$.

Gaussian channels, with $\left\langle\boldsymbol{x}_{k}\right\rangle$ regarded as a "site magnetization." Schematic pictures for the decoupling structure of the coded CDMA system are shown in figure 1. The coded CDMA channel with a multiuser detector which uses GPME (6) is, in the large-system limit, statistically equivalent to a bank of single-user scalar Gaussian channels whose noise variances are the same across users but generally different at different code symbol positions. The dependence of the variances of the scalar Gaussian channels on input symbol positions, due to effects of coding, is one unique aspect of the coded systems.

\section{LDPC-coded CDMA systems}

In this section we apply the findings obtained in the previous section to the LDPC-coded CDMA systems, focusing on a Gallager code [14], which is arguably the most common LDPC codes.

Let $N$ be the length of the original message. Gallager codes are defined by a $(M-N) \times M$ parity check matrix A (see $[8,15]$ for an elucidation of Gallager codes) in which the $\gamma$ th row weight (the number of non-zero elements in the $\gamma$ th row) is $L_{\gamma}$ and the $i$ th column weight (the number of non-zero elements in the $i$ th column) is $C_{i}$. The code rate for unbiased messages is given by $R=N / M$. If the row and column weights are the same across the rows and columns, respectively, such LDPC codes are called regular. On the other hand, irregular LDPC codes have non-constant row and/or column weights. In this paper we consider regular LDPC-coded systems for simplicity. It is straightforward to extend our formulation to cases with irregular codes.

We consider binary phase-shift-keying (BPSK) modulation and no fading effect, where codewords belong to $\{1,-1\}^{M}$. In general, the receiver knows exactly the parity check matrices, which define the prior distribution of the codewords. We therefore assume that the true prior distribution $p_{0}\left(\overrightarrow{\boldsymbol{x}}_{0}\right)$ and the postulated one $p(\overrightarrow{\boldsymbol{x}})$ are the same. We also assume that each user's code is randomly chosen from the same LDPC code ensemble. By letting the $k$ th user's parity check matrix $A_{k}$, we have 
$p_{0}\left(\overrightarrow{\boldsymbol{x}}_{0}\right)=\prod_{k} p\left(\boldsymbol{x}_{0 k} \mid \mathrm{A}_{k}\right)$ and $p(\overrightarrow{\boldsymbol{x}})=\prod_{k} p\left(\boldsymbol{x}_{k} \mid \mathrm{A}_{k}\right)$, where $p\left(\boldsymbol{x}_{k} \mid \mathrm{A}_{k}\right)$ takes a nonzero value $\left(=2^{-N}\right)$ only if $\boldsymbol{x}_{k}$ is a codeword of the code defined by $\mathrm{A}_{k}$. The GPME $\left\langle\boldsymbol{x}_{k}\right\rangle$ is rewritten as

$$
\left\langle\boldsymbol{x}_{k}\right\rangle=\sum_{\boldsymbol{x}_{k}=\{1,-1\}^{M}} \boldsymbol{x}_{k} \frac{p\left(\boldsymbol{x}_{k} \mid \mathrm{A}_{k}\right) \rho_{G}\left(\boldsymbol{z}_{k} \mid \boldsymbol{x}_{k}\right)}{\sum_{\boldsymbol{x}_{k}^{\prime}} p\left(\boldsymbol{x}_{k}^{\prime} \mid \mathrm{A}_{k}\right) \rho_{G}\left(\boldsymbol{z}_{k} \mid \boldsymbol{x}_{k}^{\prime}\right)} .
$$

The third term of the right-hand side of (18) and the order parameters (23), for the regular LDPC-coded CDMA systems, can be evaluated on the basis of the calculation given in [16]: We therefore omit details of the derivation. Denoting the third term of (18) divided by the codelength $M$ as $h_{z}$, and letting $L$ and $C$ be the row and column weights, respectively, we obtain, in the infinite codelength limit ( $M \rightarrow \infty$ with $R$ fixed), $h_{z}=\log 2-\frac{C}{L} \int \log \left(1+\prod_{l=1}^{L} u_{l}\right) \prod_{l=1}^{L}\left\{\sigma\left(u_{l}\right) \mathrm{d} u_{l}\right\}+C \int \log (1+u \hat{u}) \sigma(u) \hat{\sigma}(\hat{u}) \mathrm{d} u \mathrm{~d} \hat{u}$

$-\int \log \left[\sum_{x= \pm 1} \prod_{c=1}^{C}\left(1+x \hat{u}_{c}\right) \times \sqrt{\frac{E}{2 \pi}} \exp \left\{-\frac{E}{2}(z-x)^{2}\right\}\right]$

$\times \sqrt{\frac{E^{2}}{2 \pi F}} \exp \left\{-\frac{E^{2}}{2 F}(z-1)^{2}\right\} \bar{D} \hat{\boldsymbol{u}}_{C} \mathrm{~d} z$,

where $\bar{D} \hat{\boldsymbol{u}}_{C} \equiv \prod_{c=1}^{C}\left\{\hat{\sigma}\left(\hat{u}_{c}\right) \mathrm{d} \hat{u}_{c}\right\}$, and where the functions $\sigma(u), \hat{\sigma}(\hat{u})$ are determined by the following saddle-point equations, under the RS ansatz,

$$
\begin{aligned}
\sigma(u)=\int \delta\left(u-\tanh \left(E z+\sum_{c=1}^{C-1} \tanh ^{-1} \hat{u}_{c}\right)\right) \\
\times \sqrt{\frac{E^{2}}{2 \pi F}} \exp \left\{-\frac{E^{2}}{2 F}(z-1)^{2}\right\} \bar{D} \hat{\boldsymbol{u}}_{C-1} \mathrm{~d} z, \\
\hat{\sigma}(\hat{u})=\int \delta\left(\hat{u}-\prod_{l=1}^{L-1} \hat{u}_{l}\right) \prod_{l=1}^{L-1}\left\{\sigma\left(u_{l}\right) \mathrm{d} u_{l}\right\},
\end{aligned}
$$

and where the order parameters $\left\{E^{i}, F^{i}, m^{i}, q^{i}\right\}$ do not depend on the symbol positions in the regular LDPC code setting and are given, dropping the index $i$, by

$$
\begin{aligned}
& E=\int \bar{\rho}_{0}^{\prime}\left(y-\sqrt{\frac{\beta m^{2}}{q}} s\right) \frac{\bar{\rho}^{\prime}(y-\sqrt{\beta q} s)}{\bar{\rho}(y-\sqrt{\beta q} s)} D s \mathrm{~d} y, \\
& F=\int \bar{\rho}_{0}\left(y-\sqrt{\frac{\beta m^{2}}{q}} s\right)\left(\frac{\bar{\rho}^{\prime}(y-\sqrt{\beta q} s)}{\bar{\rho}(y-\sqrt{\beta q} s)}\right)^{2} D s \mathrm{~d} y, \\
& m=\int \tanh \left(E z+\sum_{c} \tanh ^{-1} \hat{u}_{c}\right) \sqrt{\frac{E^{2}}{2 \pi F}} \exp \left\{-\frac{E^{2}}{2 F}(z-1)^{2}\right\} \bar{D} \hat{\boldsymbol{u}}_{C} \mathrm{~d} z, \\
& q=\int \tanh ^{2}\left(E z+\sum_{c} \tanh ^{-1} \hat{u}_{c}\right) \sqrt{\frac{E^{2}}{2 \pi F}} \exp \left\{-\frac{E^{2}}{2 F}(z-1)^{2}\right\} \bar{D} \hat{\boldsymbol{u}}_{C} \mathrm{~d} z .
\end{aligned}
$$

Note that $r_{0}^{i}=r^{i}=1$ in $(23 c)$ because $x_{0}^{i}$ and $x^{i}$ take values in $\{1,-1\}$ in the present setting. 


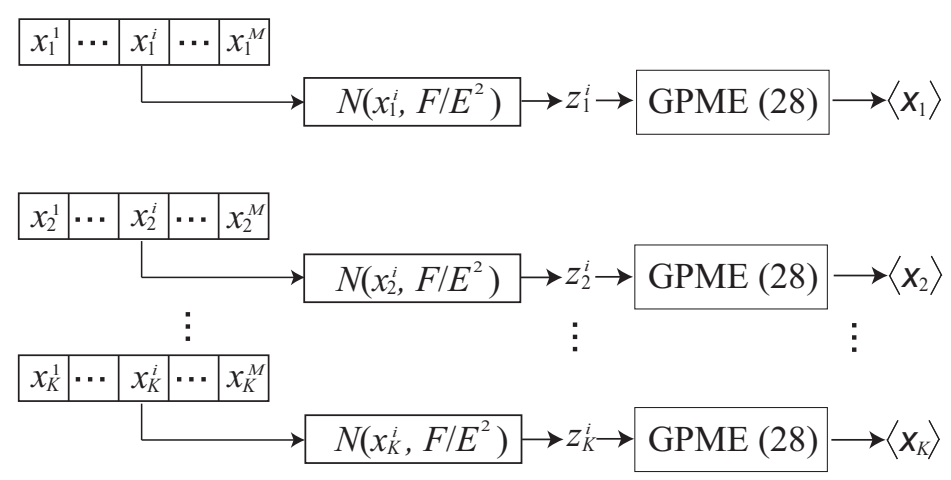

Figure 2. Decoupling structure of the regular LDPC-coded CDMA systems. The variances of the Gaussian channels take the same value, independent of users and symbol positions.

By substituting the result (29) into (18), the maximum spectral efficiency per symbol for the regular LDPC-coded CDMA system is obtained. By particularizing the channel noise of our model to additive white Gaussian noise (AWGN), our result is reduced, as it should be, to that of replica analysis of the regular LDPC-coded CDMA system over AWGN channel, presented in [17]. The mean-field description, i.e., the decoupling structure for the regular LDPC-coded CDMA systems, is schematically shown in figure 2. Since the order parameters $E^{i}, F^{i}$ do not depend on code symbol positions, in contrast to the general mean-field description of coded CDMA systems given in section 3, the regular LDPC-coded CDMA systems with GPME (28) are decoupled into a bank of single-user Gaussian channels whose variances are the same independently of the code symbol positions. This independence is due to statistical uniformity of code symbol positions in the regular LDPC code ensemble.

As shown in [17], when the row weight is not so large, the regular LDPC-coded CDMA system has two kinds of phase transitions, the decoding threshold and the information-theoretic threshold, just as those found in statistical mechanical analysis of single-user LDPC codes $[8,15,16]$. The former is also called the dynamical transition point, and the only stable solution is the perfect ferromagnetic solution when noise level is smaller than that, while the suboptimal ferromagnetic solution appears when noise level exceeds the point. The latter transition point is termed the thermodynamical transition point, at which the free energies of the above-mentioned solutions become equal, and over which the suboptimal ferromagnetic solution is dominant. Therefore, the thermodynamical transition point determines the theoretical limitation, up to which the performance could be achieved if we were given infinite computational time.

From the decoupling structure (see figure 2), one can regard that each user in the LDPC-coded CDMA system is utilizing separately from other users the single-user LDPC code over AWGN channel where the true noise variance is $\Sigma_{0}=F / E^{2}$ and the noise variance postulated at the receiver is $\Sigma=1 / E$. Assuming an LDPC-coded CDMA system over AWGN channel, and letting $\sigma_{0}^{2}$ and $\sigma^{2}$ be the true and the postulated noise variances of the AWGN channel, this observation leads to a relationship between the noise variances $\left(\sigma_{0}^{2}, \sigma^{2}\right)$ of the original LDPC-coded CDMA system over AWGN 
channel and the noise variances $\left(\Sigma_{0}, \Sigma\right)$ of the single-user LDPC code over AWGN channel. According to the relationship, one can establish correspondence between the decoding threshold of the regular LDPC-coded CDMA system over AWGN channel and that of the single-user LDPC code over AWGN channel. A numerical example of this correspondence of the decoding thresholds is shown in figure 3 , where we focus on the matched case $p_{0}(\overrightarrow{\boldsymbol{y}} \mid \overrightarrow{\boldsymbol{x}})=p(\overrightarrow{\boldsymbol{y}} \mid \overrightarrow{\boldsymbol{x}})$ for simplicity, thereby showing the relationship between $\sigma_{0}^{2}=\sigma^{2}$ and $\Sigma_{0}=\Sigma$. On the other hand, the thermodynamical transition points of the regular LDPC-coded CDMA system and the single-user LDPC code over AWGN channel do not correspond; because the perfect ferromagnetic solution and the suboptimal ferromagnetic solution of the regular LDPC-coded CDMA system at thermodynamical transition point are respectively mapped onto a ferromagnetic solution and a suboptimal ferromagnetic solution of the single-user LDPC code over AWGN channel, which, however, are with different noise variances.

For the mismatched cases where $p_{0}(\overrightarrow{\boldsymbol{y}} \mid \overrightarrow{\boldsymbol{x}})$ and $p(\overrightarrow{\boldsymbol{y}} \mid \overrightarrow{\boldsymbol{x}})$ are not equal, one can obtain the correspondence of the decoding thresholds exactly in the same way, on the basis of the relationship between $\left(\Sigma_{0}, \Sigma\right)$ and $\left(\sigma_{0}^{2}, \sigma^{2}\right)$.

Given a regular LDPC-coded CDMA system, one can determine analytically the maximum load the system can accommodate, which is given in the matched case. Figure 4 (a) shows the decoding thresholds and the thermodynamical transition points of a regular LDPC-coded CDMA system with AWGN channel. Figure 4 (b) shows the maximum spectral efficiencies of the system, achieved at the maximum loads determined by the two transition points for given signal-to-noise ratios $E_{b} / N_{0}=1 /\left(2 R \sigma_{0}^{2}\right)$. It can be seen that the theoretically achievable spectral efficiency of this system comes close to the single-user AWGN capacity [11], which is an upper bound of the spectral efficiency of multiple-access systems.

\section{Conclusions}

We have presented the mean field theory of the coded CDMA systems, in which we have obtained the analytical expression of the maximum spectral efficiency, derived on the basis of the relation between the free energy of the coded CDMA system and the mutual information. The mean-field description of the coded CDMA system with a multiuser detector based on GPME (6) has been given by a bank of single-user Gaussian channels which in general take different variances at different code symbol positions. By utilizing the result obtained for the general coded CDMA systems, the mean-field description of the regular LDPC-coded CDMA system has been characterized in terms of a bank of Gaussian channels whose variances are the same independently of code symbol positions. Using this mean-field description, we have established the correspondence between the decoding threshold of the regular LDPC-coded CDMA system and that of the single-user LDPC code over a Gaussian channel.

It is known that CDMA and MIMO systems can be modeled by a linear vector channel. Extension of our analysis to coded vector channel systems is the subject of future work. 


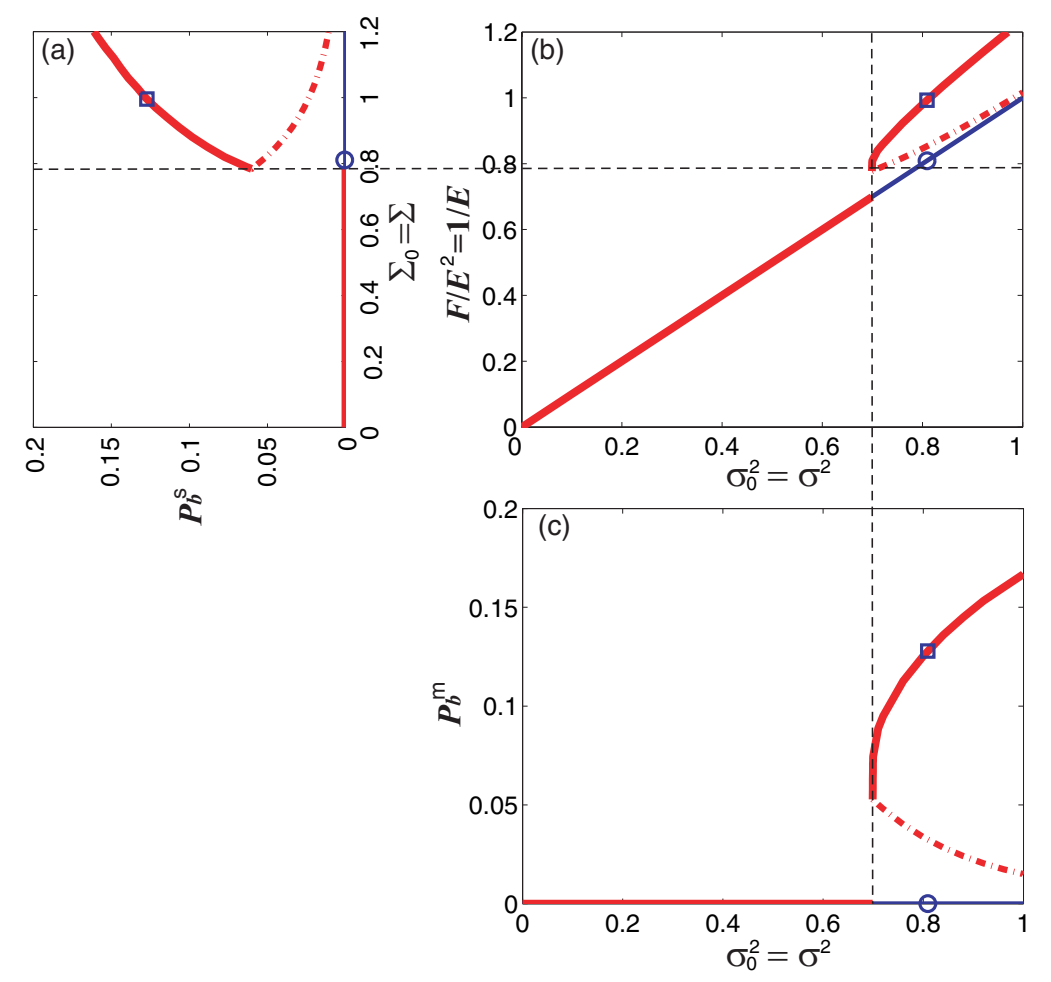

Figure 3. Relationship between the decoding threshold of a regular LDPC-coded CDMA system with AWGN channel and that of the LDPC code over AWGN channel, in the matched case. $(C, L)=(3,6)$ for both of them. (a) Bit error rate $P_{b}^{\mathrm{s}}$ of the single-user LDPC code over AWGN channel with noise variance $\Sigma_{0}=\Sigma$ (where $\Sigma_{0}$ and $\Sigma$ denote true and postulated variances, respectively, in the single-user LDPC code over AWGN channel), (b) Noise variance of the decoupled system, $F / E^{2}$, versus actual noise variance $\sigma_{0}^{2}=\sigma^{2}$ (where $\sigma_{0}^{2}$ and $\sigma^{2}$ denote true and postulated variances, respectively), and (c) Bit error rate $P_{b}^{\mathrm{m}}$ of the LDPC-coded CDMA system. The thick solid line in (a) is obtained by evaluating numerically the saddle-point equations derived in [8] with AWGN (variance: $\Sigma_{0}=\Sigma$ ). Those in (b) and (c) are given by evaluating numerically the saddle-point equations (30) and (31) with AWGN (variance: $\sigma_{0}^{2}=\sigma^{2}$ ) and $\beta=0.5$. The dot-dashed lines denote unstable solutions, which are not obtained numerically, but are schematically drawn for better understanding. At the decoding threshold of the LDPC-coded CDMA system (marked by the vertical dashed line), the variance of the Gaussian channel given in the decoupling structure, $F / E^{2}$, corresponds to the decoding threshold, characterized by $\Sigma_{0}$, of the single-user LDPC code over AWGN channel (marked by the horizontal dashed line). In (b) and (c), the circles $O$ and squares $\square$ denote, respectively, the perfect ferromagnetic solution and the suboptimal ferromagnetic solution at the thermodynamical transition point. The circle $\mathrm{O}$ and square $\square$ in (a), which are mapped from (b), are located at different noise variances.

\section{Acknowledgments}

TT acknowledges support from the Grant-in-Aid for Scientific Research on Priority Areas, the Ministry of Education, Culture, Sports, Science and Technology, Japan (no. 18079010). DS acknowledges support from EVERGROW, IP No. 1935 in FP6 of the EU and EPSRC grant EP/E049516/1. 

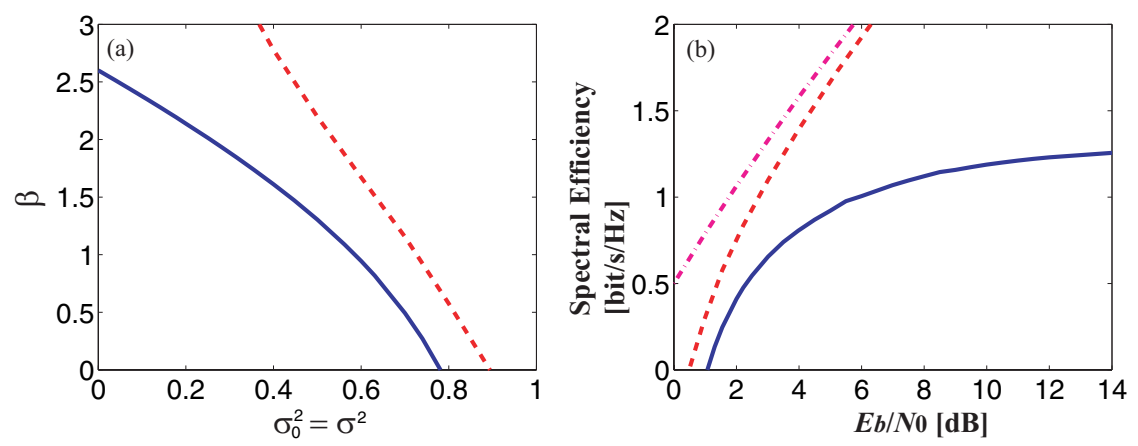

Figure 4. (a) The maximum loads $\beta$ corresponding to decoding threshold (solid line) and thermodynamical transition point (dashed line) of the $(C, L)=(3,6)$ regular LDPC-coded CDMA system with AWGN channel. (b) Spectral efficiencies, achieved at the maximum loads corresponding to the two transition points shown in (a), versus signal-to-noise ratio $E_{b} / N_{0}$. The single-user AWGN capacity [11] (dot-dashed line) is also shown.

\section{Appendix A. Evaluation of the conditional differential entropy conditioned on the spreading sequences}

In this appendix we evaluate the conditional differential entropy $h$, by basically following the procedures in $[2,3]$. We denote the original channel input and the replicated channel inputs as $\overrightarrow{\boldsymbol{x}}_{0}$ and $\left\{\overrightarrow{\boldsymbol{x}}_{a} ; a=1, \ldots, n\right\}$, respectively. We let $\boldsymbol{x}_{a}^{i}=\left(x_{a 1}^{i}, \ldots, x_{a K}^{i}\right)$ for $a=0, \ldots, n$. Assuming that $n$ is a positive integer, one obtains

$$
\Xi_{n}=\int\left[\int \mathrm{E}_{S}\left\{\prod_{a=0}^{n}\left(\prod_{i=1}^{M} \prod_{\mu=1}^{W} \rho_{a}\left(y_{\mu}^{i}-\frac{1}{\sqrt{W}} \sum_{k=1}^{K} s_{\mu k}^{i} x_{a k}^{i}\right)\right)\right\} \mathrm{d} \overrightarrow{\boldsymbol{y}}\right] \prod_{a=0}^{n}\left[p_{a}\left(\overrightarrow{\boldsymbol{x}}_{a}\right) \mathrm{d} \overrightarrow{\boldsymbol{x}}_{a}\right],
$$

where $\rho_{a}(\cdot)=\rho(\cdot)$ and $p_{a}\left(\overrightarrow{\boldsymbol{x}}_{a}\right)=p\left(\overrightarrow{\boldsymbol{x}}_{a}\right)$ for $a=1, \ldots, n$.

Independence of the spreading sequences with respect to index $\mu$ allows us to rewrite $\Xi_{n}$ as

$$
\Xi_{n}=\int e^{W \mathcal{G}} \prod_{a=0}^{n}\left[p_{a}\left(\overrightarrow{\boldsymbol{x}}_{a}\right) \mathrm{d} \overrightarrow{\boldsymbol{x}}_{a}\right]
$$

where

$$
\begin{aligned}
e^{\mathcal{G}} & =\int \mathrm{E}_{s}\left\{\prod_{a=0}^{n}\left(\prod_{i=1}^{M} \rho_{a}\left(y^{i}-\sqrt{\beta} v_{a}^{i}\right)\right)\right\} \prod_{i} \mathrm{~d} y^{i}, \\
v_{a}^{i} & =v_{a}^{i}\left(\boldsymbol{s}^{i}\right)=\frac{1}{\sqrt{K}} \sum_{k=1}^{K} s_{k}^{i} x_{a k}^{i}, \quad a=0, \ldots, n .
\end{aligned}
$$

Here, $\boldsymbol{s}^{i}=\left(s_{1}^{i}, \ldots, s_{K}^{i}\right)$ and $\mathrm{E}_{s}\{\cdots\}$ denotes averaging over $\left\{\boldsymbol{s}^{i} ; i=1, \ldots, M\right\}$.

Since $v_{a}^{i}$ is a sum of the i.i.d. random variables of $\boldsymbol{s}^{i}, \boldsymbol{v}^{i}=\left(v_{1}^{i}, v_{2}^{i}, \ldots, v_{n}^{i}\right)$ conditioned on $\left\{\boldsymbol{x}_{a}^{i} ; a=0, \ldots, n\right\}$ follows, in the large-system limit, a multivariate Gaussian distribution with mean $\mathbf{0}$ and covariance matrix $Q^{i}=\left(Q_{a b}^{i}\right)$ with

$$
Q_{a b}^{i}=\frac{1}{K} \sum_{k=1}^{K} x_{a k}^{i} x_{b k}^{i},
$$


via the central limit theorem $\left(\boldsymbol{v}^{i}\right.$ and $\boldsymbol{v}^{j}(i \neq j)$ are independent as the spreading sequences are also independent with respect to coded symbol indices $i, j$ ). The expectation $\mathrm{E}_{s}\{\cdots\}$ in (A.3) therefore reduces to averaging over Gaussian random variables $\boldsymbol{v}^{i}(i=1, \ldots, M)$, yielding

$$
e^{\mathcal{G}(\boldsymbol{Q})}=\exp \left(\sum_{i=1}^{M} g\left(Q^{i}\right)\right)
$$

where $\boldsymbol{Q}=\left(Q^{1}, Q^{2}, \ldots, Q^{M}\right)$ and $\exp \left(g\left(Q^{i}\right)\right)$ is given by

$$
e^{g\left(Q^{i}\right)}=\int \mathrm{E}_{\boldsymbol{v}^{i}}\left\{\rho_{0}\left(y^{i}-\sqrt{\beta} v_{0}^{i}\right) \prod_{a=1}^{n} \rho\left(y^{i}-\sqrt{\beta} v_{a}^{i}\right)\right\} \mathrm{d} y^{i} .
$$

Since $e^{\mathcal{G}(\boldsymbol{Q})}$ depends on $\left\{\overrightarrow{\boldsymbol{x}}_{a} ; a=0, \ldots, n\right\}$ only via $\boldsymbol{Q}$, we obtain

$$
\Xi_{n}=\int e^{W \mathcal{G}(\boldsymbol{Q})} \mu_{K}(\boldsymbol{Q}) \mathrm{d} \boldsymbol{Q},
$$

where $\mathrm{d} \boldsymbol{Q}=\prod_{i} \prod_{a \leq b} \mathrm{~d} Q_{a b}^{i}$, and where $\mu_{K}(\boldsymbol{Q})$ is the probability measure of $\boldsymbol{Q}$, which is defined as

$$
\mu_{K}(\boldsymbol{Q})=\int \prod_{a=0}^{n} p_{a}\left(\overrightarrow{\boldsymbol{x}}_{a}\right) \prod_{i=1}^{M} \prod_{a \leq b} \delta\left(\sum_{k=1}^{K} x_{a k}^{i} x_{b k}^{i}-K Q_{a b}^{i}\right) \prod_{a=0}^{n} \mathrm{~d} \overrightarrow{\boldsymbol{x}}_{a} .
$$

Assuming that the measure $\mu_{K}(\boldsymbol{Q})$ satisfies the large-deviation principle as $K \rightarrow \infty$ with a rate function $\Lambda(\boldsymbol{Q})$, we have the heuristic formula $\mu_{K}(\boldsymbol{Q}) \approx e^{-K \Lambda(\boldsymbol{Q})}$ in the large-system limit [18], which allows us to apply the saddle-point method to obtain

$$
\lim _{K \rightarrow \infty} \frac{1}{K} \log \Xi_{n}=\sup _{\boldsymbol{Q}}\left[\beta^{-1} \mathcal{G}(\boldsymbol{Q})-\Lambda(\boldsymbol{Q})\right] .
$$

The rate function $\Lambda(\boldsymbol{Q})$ is given by the Legendre transform of the cumulant generating function $\phi(\tilde{\boldsymbol{Q}})$, as

$$
\Lambda(\boldsymbol{Q})=\sup _{\tilde{\boldsymbol{Q}}}[\boldsymbol{Q} \cdot \tilde{\boldsymbol{Q}}-\phi(\tilde{\boldsymbol{Q}})]
$$

where $\boldsymbol{Q} \cdot \tilde{\boldsymbol{Q}}=\sum_{i} \sum_{a \leq b} Q_{a b}^{i} \tilde{Q}_{a b}^{i}$, and $\phi(\tilde{\boldsymbol{Q}})$ is defined by the following formula, assuming that the limit exists:

$\phi(\tilde{\boldsymbol{Q}})=\lim _{K \rightarrow \infty} \frac{1}{K} \log \left\{\int \prod_{a=0}^{n} p_{a}\left(\overrightarrow{\boldsymbol{x}}_{a}\right) \exp \left(\sum_{i=1}^{M} \sum_{a \leq b} \tilde{Q}_{a b}^{i} \sum_{k=1}^{K} x_{a k}^{i} x_{b k}^{i}\right) \prod_{a=0}^{n} \mathrm{~d} \overrightarrow{\boldsymbol{x}}_{a}\right\}$.

Summarizing the above results, one obtains

$$
\begin{aligned}
\lim _{K \rightarrow \infty} \frac{1}{K} \log \Xi_{n} & =\sup _{\boldsymbol{Q}} \inf _{\tilde{\boldsymbol{Q}}}\left[\beta^{-1} \mathcal{G}(\boldsymbol{Q})-\boldsymbol{Q} \cdot \tilde{\boldsymbol{Q}}+\phi(\tilde{\boldsymbol{Q}})\right] \\
& =\sup _{\boldsymbol{Q}} \inf _{\tilde{\boldsymbol{Q}}}\left[\beta^{-1} \sum_{i} g\left(Q^{i}\right)-\boldsymbol{Q} \cdot \tilde{\boldsymbol{Q}}+\phi(\tilde{\boldsymbol{Q}})\right] .
\end{aligned}
$$

Now let us make the replica symmetry $(\mathrm{RS})$ assumption to proceed further, on the basis of which we let, for $a \neq b, a \neq 0, b \neq 0$,

$$
\begin{aligned}
& Q_{00}^{i}=r_{0}^{i}, \quad Q_{a a}^{i}=r^{i}, \quad Q_{0 a}^{i}=m^{i}, \quad Q_{a b}^{i}=q^{i}, \\
& \tilde{Q}_{00}^{i}=\frac{G_{0}^{i}}{2}, \quad \tilde{Q}_{a a}^{i}=\frac{G^{i}}{2}, \quad \tilde{Q}_{0 a}^{i}=E^{i}, \quad \tilde{Q}_{a b}^{i}=F^{i} .
\end{aligned}
$$


Using expressions of the Gaussian random variables $\left\{v_{a}^{i}\right\}$ in terms of standard Gaussian random variables $n_{0}^{i}, n_{a}^{i}, t^{i}$,

$$
\begin{aligned}
& v_{0}^{i}=\sqrt{r_{0}^{i}-\frac{\left(m^{i}\right)^{2}}{q^{i}}} n_{0}^{i}+\sqrt{\frac{\left(m^{i}\right)^{2}}{q^{i}}} t^{i}, \\
& v_{a}^{i}=\sqrt{r^{i}-q^{i}} n_{a}^{i}+\sqrt{q^{i}} t^{i}, \quad a=1, \ldots, n,
\end{aligned}
$$

we obtain, under the RS ansatz,

$$
e^{g\left(Q^{i}\right)}=\int \bar{\rho}_{0}\left(y^{i}-\sqrt{\frac{\beta\left(m^{i}\right)^{2}}{q^{i}}} t^{i}\right)\left[\bar{\rho}\left(y^{i}-\sqrt{\beta q^{i}} t^{i}\right)\right]^{n} D t^{i} \mathrm{~d} y^{i},
$$

where $\bar{\rho}_{0}(\cdot)$ and $\bar{\rho}(\cdot)$ are defined by (19) and (20), respectively. Using the HubbardStratonovich transform

$$
e^{x^{2}}=\sqrt{\frac{\eta}{2 \pi}} \int \exp \left[-\frac{\eta}{2} z^{2}+\sqrt{2 \eta} x z\right] \mathrm{d} z
$$

one finds that

$$
\begin{aligned}
\phi(\tilde{\boldsymbol{Q}})=\lim _{K \rightarrow \infty} & \frac{1}{K} \log \left[\int\left\{\int p_{0}\left(\overrightarrow{\boldsymbol{x}}_{0}\right) \times e^{\sum_{k, i} \frac{G_{0}^{i}}{2}\left(x_{0 k}^{i}\right)^{2}} \prod_{k} \rho_{G 0}\left(\boldsymbol{z}_{k} \mid \boldsymbol{x}_{0 k}\right) \mathrm{d} \overrightarrow{\boldsymbol{x}}_{0}\right\}\right. \\
& \left.\times\left\{\int p(\overrightarrow{\boldsymbol{x}}) \times e^{\sum_{k, i} \frac{G^{i}-F^{i}+E^{i}}{2}\left(x_{k}^{i}\right)^{2}} \prod_{k} \rho_{G}\left(\boldsymbol{z}_{k} \mid \boldsymbol{x}_{k}\right) \mathrm{d} \overrightarrow{\boldsymbol{x}}\right\}^{n} e^{\frac{n}{2} \sum_{k, i} E^{i}\left(z_{k}^{i}\right)^{2}} \mathrm{~d} \overrightarrow{\boldsymbol{z}}\right] \\
& +\frac{n}{2} \sum_{i} \log \frac{2 \pi}{E^{i}},
\end{aligned}
$$

where $\rho_{G 0}\left(\boldsymbol{z}_{k} \mid \boldsymbol{x}_{0 k}\right)$ and $\rho_{G}\left(\boldsymbol{z}_{k} \mid \boldsymbol{x}_{k}\right)$ are given by (21) and (22), respectively. $\boldsymbol{Q} \cdot \tilde{\boldsymbol{Q}}$ is given, under the RS ansatz, by

$$
\boldsymbol{Q} \cdot \tilde{\boldsymbol{Q}}=\sum_{i}\left(r_{0}^{i} \frac{G_{0}^{i}}{2}+n r^{i} \frac{G^{i}}{2}+n m^{i} E^{i}+\frac{n(n-1)}{2} q^{i} F^{i}\right) .
$$

Saddle-point equations of the order parameters are derived from the extremum conditions in (A.13). The saddle-point equations for $\left\{G_{0}^{i}, G^{i}\right\}$ are, in the limit $n \rightarrow 0$,

$$
\begin{aligned}
& G_{0}^{i}=0, \\
& G^{i}=\int \bar{\rho}_{0}\left(y^{i}-\sqrt{\frac{\beta\left(m^{i}\right)^{2}}{q^{i}}} t^{i}\right) \frac{\bar{\rho}^{\prime \prime}\left(y^{i}-\sqrt{\beta q^{i}} t^{i}\right)}{\bar{\rho}\left(y^{i}-\sqrt{\beta q^{i}} t^{i}\right)} D t^{i} \mathrm{~d} y^{i} .
\end{aligned}
$$

Those of $\left\{E^{i}, F^{i}, r_{0}^{i}, r^{i}, m^{i}, q^{i}\right\}$ are given by $(23 a)-(23 d)$. By observing that

$$
G^{i}+E^{i}-F^{i}=0
$$

holds for additive channel noise, and that

$$
\lim _{K \rightarrow \infty} \frac{1}{K} \int \frac{1}{2} \sum_{k, i} E^{i}\left(z_{k}^{i}\right)^{2} p_{0}\left(\overrightarrow{\boldsymbol{x}}_{0}\right) \prod_{k} \rho_{G 0}\left(\boldsymbol{z}_{k} \mid \boldsymbol{x}_{0 k}\right) \mathrm{d} \overrightarrow{\boldsymbol{z}} \mathrm{d} \overrightarrow{\boldsymbol{x}}_{0}=\sum_{i}\left(\frac{F^{i}}{2 E^{i}}+\frac{E^{i}}{2} r_{0}^{i}\right),
$$

the final form of $h(18)$ is obtained. 


\section{Appendix B. Derivation of the decoupling structure of the coded CDMA systems}

We assume that the joint distributions $p\left(\left[\boldsymbol{x}_{k}\right], \boldsymbol{x}_{0 k} \mid S\right)$ and $p\left(\left\langle\boldsymbol{x}_{k}\right\rangle, \boldsymbol{x}_{0 k}\right)$ in (27) are uniquely determined by their joint moments, on the basis of which we will show that these distributions are asymptotically statistically equivalent with each other by showing that their joint moment sequences coincide with each other in the large-system limit. We also assume the self-averaging property of the joint moments of $p\left(\left[\boldsymbol{x}_{k}\right], \boldsymbol{x}_{0 k} \mid S\right)$ over the random spreading sequences in the large-system limit. It is sufficient to show that the expectation of any arbitrary joint moment of $p\left(\left[\boldsymbol{x}_{k}\right], \boldsymbol{x}_{0 k} \mid S\right)$ over the random spreading sequences converges to the corresponding joint moment of $p\left(\left\langle\boldsymbol{x}_{k}\right\rangle, \boldsymbol{x}_{0 k}\right)$, in the largesystem limit and under the random spreading assumption. The joint moments are evaluated in a similar way to the evaluation of the conditional differential entropy in Appendix A, using the replica method.

Given a class $\mathscr{K}$ which accommodates $K_{1}=\varepsilon_{1} K$ users for $0<\varepsilon_{1}<1$, we consider a set $\mathscr{X}=\left\{\boldsymbol{x}_{a k} ; a=0, \ldots, n, k \in \mathscr{K}\right\}$. We define a function $f(\mathscr{X})$ as

$$
f(\mathscr{X})=\sum_{k \in \mathscr{K}} \prod_{i=1}^{M}\left\{\left(x_{0 k}^{i}\right)^{\lambda^{i}} \prod_{b^{i} \in \alpha^{i}}\left(x_{b^{i} k}^{i}\right)\right\},
$$

where $\alpha^{i}$ is a mutually disjoint subset composed of $\mu^{i}$ different replicas for $i=1, \ldots, M$. For a fixed $\varepsilon_{1}$, we now define, using the function $f(\mathscr{X})$, a quantity similar to the conditional differential entropy (15)

$$
\tilde{h}=\lim _{K \rightarrow \infty} \frac{1}{K_{1}} \log \tilde{\Xi}_{n}
$$

where $\tilde{\Xi}_{n}$ is given by

$\tilde{\Xi}_{n}=\int \prod_{a=0}^{n} p_{a}\left(\overrightarrow{\boldsymbol{x}}_{a}\right)\left[\int \mathrm{E}_{S}\left\{\prod_{a=0}^{n} p_{a}\left(\overrightarrow{\boldsymbol{y}} \mid \overrightarrow{\boldsymbol{x}}_{a}\right) \exp (\omega f(\mathscr{X}))\right\} \mathrm{d} \overrightarrow{\boldsymbol{y}}\right] \prod_{a=0}^{n} \mathrm{~d} \overrightarrow{\boldsymbol{x}}_{a}$.

We thus obtain joint moments of $p\left([\overrightarrow{\boldsymbol{x}}], \overrightarrow{\boldsymbol{x}}_{0} \mid S\right)$ as

$\left.\lim _{n \rightarrow 0} \frac{\partial \tilde{h}}{\partial \omega}\right|_{\omega=0}=\lim _{K \rightarrow \infty} \frac{1}{K_{1}} \sum_{k \in \mathscr{K}} \mathrm{E}_{S}\left[\int p_{0}\left(\overrightarrow{\boldsymbol{x}}_{0}\right) p_{0}\left(\overrightarrow{\boldsymbol{y}} \mid \overrightarrow{\boldsymbol{x}}_{0}\right) \prod_{i}\left\{\left(x_{0 k}^{i}\right)^{\lambda^{i}}\left[x_{k}^{i}\right]^{\mu^{i}}\right\} \mathrm{d} \overrightarrow{\boldsymbol{y}} \mathrm{d} \overrightarrow{\boldsymbol{x}}_{0}\right]$.

Evaluation of (B.2) can be done as follows. By assuming existence of the limit $\tilde{\phi}(\tilde{\boldsymbol{Q}})$

$=\lim _{K \rightarrow \infty} \frac{1}{K} \log \left\{\int \prod_{a=0}^{n} p_{a}\left(\overrightarrow{\boldsymbol{x}}_{a}\right) \exp \left(\omega f(\mathscr{X})+\sum_{i=1}^{M} \sum_{a \leq b} \tilde{Q}_{a b}^{i} \sum_{k=1}^{K} x_{a k}^{i} x_{b k}^{i}\right) \prod_{a=0}^{n} \mathrm{~d} \overrightarrow{\boldsymbol{x}}_{a}\right\}$,

(B.2) is rewritten as

$$
\tilde{h}=\frac{1}{\varepsilon_{1}} \sup _{\boldsymbol{Q}} \inf _{\tilde{\boldsymbol{Q}}}\left[\beta^{-1} \mathcal{G}(\boldsymbol{Q})-\boldsymbol{Q} \cdot \tilde{\boldsymbol{Q}}+\tilde{\phi}(\tilde{\boldsymbol{Q}})\right] .
$$

Therefore we obtain

$$
\left.\frac{\partial \tilde{h}}{\partial \omega}\right|_{\omega=0}=\frac{1}{\varepsilon_{1}} \times\left.\frac{\partial \tilde{\phi}(\tilde{\boldsymbol{Q}})}{\partial \omega}\right|_{\omega=0},
$$


where

$$
\left.\frac{\partial \tilde{\phi}(\tilde{\boldsymbol{Q}})}{\partial \omega}\right|_{\omega=0}=\lim _{K \rightarrow \infty} \frac{1}{K} \frac{\left\{\int \prod_{a} p\left(\overrightarrow{\boldsymbol{x}}_{a}\right) f(\mathscr{X}) \exp \left(\sum_{i} \sum_{a \leq b} \tilde{Q}_{a b}^{i} \sum_{k} x_{a k}^{i} x_{b k}^{i}\right) \prod_{a} \mathrm{~d} \overrightarrow{\boldsymbol{x}}_{a}\right\}}{\left\{\int \prod_{a} p\left(\overrightarrow{\boldsymbol{x}}_{a}\right) \exp \left(\sum_{i} \sum_{a \leq b} \tilde{Q}_{a b}^{i} \sum_{k} x_{a k}^{i} x_{b k}^{i}\right) \prod_{a} \mathrm{~d} \overrightarrow{\boldsymbol{x}}_{a}\right\}} .
$$

By evaluating (B.5) under the RS ansatz (A.14), the right-hand side of (B.7) in the limit $n \rightarrow 0$ is given by

$$
\lim _{n \rightarrow 0}\left\{\frac{1}{\varepsilon_{1}} \times\left.\frac{\partial \tilde{\phi}(\tilde{\boldsymbol{Q}})}{\partial \omega}\right|_{\omega=0}\right\}=\lim _{K \rightarrow \infty} \frac{1}{K_{1}} \sum_{k \in \mathscr{K}}\left\langle\left\langle\prod_{i}\left(x_{0 k}^{i}\right)^{\lambda^{i}}\left\langle x_{k}^{i}\right\rangle^{\mu^{i}}\right\rangle\right\rangle,
$$

where $\langle\cdots\rangle$ and $\langle\langle\cdots\rangle\rangle$ are defined by $(24)$ and (25), respectively. From (B.4) and (B.9) we obtain

$$
\begin{aligned}
& \lim _{K \rightarrow \infty} \frac{1}{K_{1}} \sum_{k \in \mathscr{K}} \mathrm{E}_{S}\left[\int p_{0}\left(\overrightarrow{\boldsymbol{x}}_{0}\right) p_{0}\left(\overrightarrow{\boldsymbol{y}} \mid \overrightarrow{\boldsymbol{x}}_{0}\right) \prod_{i}\left\{\left(x_{0 k}^{i}\right)^{\lambda^{i}}\left[x_{k}^{i}\right]^{\mu^{i}}\right\} \mathrm{d} \overrightarrow{\boldsymbol{y}} \mathrm{d} \overrightarrow{\boldsymbol{x}}_{0}\right] \\
& =\lim _{K \rightarrow \infty} \frac{1}{K_{1}} \sum_{k \in \mathscr{K}}\left\langle\left\langle\prod_{i}\left(x_{0 k}^{i}\right)^{\lambda^{i}}\left\langle x_{k}^{i}\right\rangle^{\mu^{i}}\right\rangle\right\rangle .
\end{aligned}
$$

Letting $\varepsilon_{1} \rightarrow 0$, so $K_{1} \rightarrow 1$ (whose justification is not discussed here, but will be given on the basis of an argument in [13]), we have, in the large-system limit $K \rightarrow \infty$,

$\mathrm{E}_{S}\left[\int p_{0}\left(\overrightarrow{\boldsymbol{x}}_{0}\right) p_{0}\left(\overrightarrow{\boldsymbol{y}} \mid \overrightarrow{\boldsymbol{x}}_{0}\right) \prod_{i}\left\{\left(x_{0 k}^{i}\right)^{\lambda^{i}}\left[x_{k}^{i}\right]^{\mu^{i}}\right\} \mathrm{d} \overrightarrow{\boldsymbol{y}} \mathrm{d} \overrightarrow{\boldsymbol{x}}_{0}\right]=\left\langle\left\langle\prod_{i}\left(x_{0 k}^{i}\right)^{\lambda^{i}}\left\langle x_{k}^{i}\right\rangle^{\mu^{i}}\right\rangle\right\rangle$,

which means that the joint distributions $p\left(\left[\boldsymbol{x}_{k}\right], \boldsymbol{x}_{0 k} \mid S\right)$ and $p\left(\left\langle\boldsymbol{x}_{k}\right\rangle, \boldsymbol{x}_{0 k}\right)$ share the same joint moments under random spreading and in the large-system limit.

\section{References}

[1] Tanaka T 2001 Europhys. Lett. 54 540-546

[2] Tanaka T 2002 IEEE Trans. Info. Theory 48 2888-2910

[3] Guo D and Verdú S 2005 IEEE Trans. Info. Theory 51 1983-2010

[4] Moustakas A L, Simon S H and Sengupta A M 2003 IEEE Trans. Info. Theory 49 2545-2561

[5] Müller R R 2003 IEEE Trans. Sig. Proc. 51 2821-2828

[6] Alamino R C and Saad D 2007 J. Phys. A: Math. Theor. 40 12259-12279

[7] Kasera S and Narang N $20053 G$ Mobile Networks (New York, USA: McGraw-Hill)

[8] Tanaka T and Saad D 2003 J. Phys. A: Math. Gen. 36 11143-11157

[9] Verdú S 1998 Multiuser Detection (Cambridge, U.K.: Cambridge Univ. Press)

[10] Cover T M and Thomas J A 2006 Elements of Information Theory 2nd ed (Hoboken, NJ: WileyInterscience)

[11] Verdú S and Shamai (Shitz) S 1999 IEEE Trans. Info. Theory 45 622-640

[12] Tanaka T 2006 Proc. 2006 IEEE Int. Symp. Info. Theory (Seattle, WA) pp 2368-2372

[13] Tanaka T and Nakamura K 2008 Eur. Phys. J. B 10.1140/epjb/e2008-00096-x

[14] Gallager R G 1963 Low-Density Parity-Check Codes (Research Monograph) (Cambridge, MA: MIT Press)

[15] Kabashima Y and Saad D 2004 J. Phys. A: Math. Gen. 37 R1-R43

[16] Murayama T, Kabashima Y, Saad D and Vicente R 2000 Phys. Rev. E 62 1577-1591

[17] Tanaka T and Saad D 2003 Proc. 2003 IEEE Int. Symp. Info. Theory (Yokohama, Japan) p 444

[18] Dembo A and Zeitouni O 1998 Large Deviations Techniques and Applications 2nd ed (Applications of Mathematics vol 38) (New York: Springer-Verlag) 